\title{
Predictive Value of A Novel IncRNA LINC02518 in Evaluating the Prognosis of Patients With Hepatocellular Carcinoma
}

Wei Cui ( $\square$ cuiwei@gdph.org.cn )

Guangdong Provincial People's Hospital https://orcid.org/0000-0002-3356-5054

Jingzhi Huang

SYSU

Ruiqi Wang

Sun Yat-Sen University

Yu Wang

SYSU

Xiaoming Chen

GDPH

Feng Shi

GDPH

Jiaping Li

SYSU

Rongde Xu

GDPH

\section{Primary research}

Keywords: Iong non-coding RNA, hepatocellular carcinoma, prognosis, predictive value

Posted Date: August 31st, 2020

DOI: https://doi.org/10.21203/rs.3.rs-66109/v1

License: (c) (1) This work is licensed under a Creative Commons Attribution 4.0 International License.

Read Full License

Version of Record: A version of this preprint was published at Biomarkers in Medicine on September 6th, 2021. See the published version at https://doi.org/10.2217/bmm-2020-0795. 


\section{Abstract}

BACKGROUND: The potential of IncRNA in hepatocellular carcinoma (HCC) has led to promising insights in therapeutic intervention. The clinical significance of LINC02518 in HCC is unclear. This study aimed to evaluate the predictive value of a novel long non-coding RNA LINC02518 for the prognosis of patients with HCC.

METHODS: Between December 2005 and November 2011, 125 HCC patients in training group and 75 HCC patients in validation group who underwent liver surgery were involved in our study. The LINC02518 expression of HCC and corresponding non-tumor liver tissues was detected by microarray and qRT-PCR. These HCC patients were divided into high and low LINC02518 expression groups based on the threshold of the receiver operating characteristic curve. Kaplan-Meier analysis was performed to determine the prognosis of HCC patients.

RESULTS: LINC02518 expression was upregulated in paired tumor samples compared to that in corresponding non-tumor samples in two groups. The areas under the receiver operating characteristic curve for the levels of LINC02518 in the diagnosis of HCC was $0.66,95 \% \mathrm{Cl}: 0.59-0.73$. HCC patients with high LINC02518 expression had significantly worse tumor recurrence-free, metastasis-free, disease-free, and overall survival than those with low LINC02518 expression.

CONCLUSIONS: LINC02518 is negatively correlated with the prognosis of HCC and provides a promising strategy for the treatment and prognosis of HCC.

\section{Introduction}

Liver cancer is the sixth most common type of cancer in patients and the sixth cause for cancer-related mortalities (1). Hepatocellular carcinoma (HCC) is the predominant form of primary liver cancers. HCC is caused by chronic hepatitis B and C infections in Asia and Africa. Notably, HBV-related HCC accounts for $85 \%$ of the total HCC cases in China (2). Unfortunately, middle and advanced stage HCC comprises the patient population that can be attributed to the poor 5-year survival rate (1). The primary treatment strategies for HCC include molecular targeted therapy, systemic chemotherapy, hepatic arterial infusion chemotherapy, transcatheter artery chemoembolization, and immunotherapy $(1,3)$. Although previous studies have reported multiple aberrantly expressed protein-coding genes in $\mathrm{HCC}$, there is an urgent need to identify novel molecular markers that may help in the early diagnosis and risk assessment of HCC. It is imperative to understand the correlation between clinical symptoms and molecular changes in HCC to develop new diagnostic and treatment strategies against HCC and improve prognosis of diagnosed patients (4).

It has been recently reported that long non-coding RNA (IncRNA) plays a critical role in numerous biological processes, such as regulating miRNA function, transcription, translation, protein localization, controlling cell cycle, differentiation, apoptosis and DNA repair, and immune cell lineage development (57). LncRNAs show differential expression in many tumors, suggesting that these long transcripts may be 
used as specific biomarkers to diagnose and treat cancer. The potential of IncRNAs in HCC has led to promising insights in therapeutic intervention (8). Therefore, further studies are needed to explore the role of IncRNAs in HCC. This study aimed to investigate the prognostic value of the novel IncRNA LINC02518 in HCC patients that can be developed into biomarkers and identify potential targets for effective treatment.

\section{Materials And Methods}

\section{Patient cohort}

This study comprised $125 \mathrm{HCC}$ cases in training group and $75 \mathrm{HCC}$ cases in validation group having undergone radical resection between December 2005 and November 2011. HCC diagnosis was based on the criteria by the European Association for the Study of the Liver (9): histopathological analysis and positive lesion(s) detected by at least two different imaging techniques [computed tomography (CT) and magnetic resonance imaging (MRI)] or a positive lesion detected by one technique (CT or MRI) combined with a-fetoprotein (AFP) levels of $>400 \mathrm{ng} / \mathrm{ml}$. The Barcelona Clinic Liver Cancer (BCLC) and Tumor, Node, Metastasis (TNM) staging were used for categorizing the tumors $(10,11)$. The study protocol was in accordance with the ethical guidelines of the Declaration of Helsinki and approved by the ethics committee of our hospital. The requirement for informed consent was waived since this was a retrospective clinical study. The inclusion criteria were: (1) age >18 years; (2) newly diagnosed with HCC; (3) Child-Pugh classification A or B; and (4) no history of previous anticancer therapy. The exclusion criteria were: (1) diagnosis or history of other malignancies; (2) main portal vein obstruction; (3) ChildPugh C class liver function; (4) massive ascites, severe coagulation, or kidney disorders; and (5) patients with no follow-up data. No deaths occurred within 1 month of radical hepatectomy.

\section{LncRNA microarray screen and exploring the characteristics of LINC02518}

All the tissue samples were frozen at $-80^{\circ} \mathrm{C}$ and total amount and quality of RNA extracted met the experimental requirements for biochip and quantitative real-time polymerase chain reaction. Total RNA was extracted using TriZol (Invitrogen, Carlsbad, CA) according to the kit instructions. RNA quality was confirmed by formaldehyde agarose gel electrophoresis and quantified by NanoDrop ND-1000 (12). We retrospectively analyzed IncRNA expression profiles of $125 \mathrm{HCCs}$ with a custom microarray (LncRNA Expression Microarray) screened from the IncRNA database (Noncode, Genecode, LncRNAdb, Refseq) with a total of 2,412 IncRNA transcripts. The samples (HCC and adjacent normal liver tissues) were used to synthesize double-stranded cDNA that was labeled and hybridized to the LncRNA Expression Microarray according to the protocol provided (12). After hybridization, the arrays were washed, and slides were scanned using the Agilent Microarray Scanner. Raw data were extracted as pair files using Agilent Feature Extraction. A random variance model was used to identify differentially expressed genes (12). Subsequently, differential expression of IncRNAs in cancer and adjacent normal liver tissues were analyzed by the Significance Analysis of Microarrays software. The IncRNA microarray standardized data have been uploaded to the Gene Expression Omnibus Public Database (GEO) of NCBI website (National 
Center for Biotechnology Information, USA); data are available via GEO GSE72170. Among the IncRNAs, LINC02518 was identified as a novel candidate IncRNA. Also, qRT-PCR were performed to validate the LINC02518 RNA expression levels in 75 paired HCC and peripheral tissues. The primers sequences were as follows: GAPDH, forward:5'- TTGCCCTCAACGACCACTTT -3';reverse: 5'- CGCCAGACCCTGCACTTTTT -3'; LINC02518, forward:5'- AGACTTCATGCACACTCATCTCA-3';reverse: 5'- TGGCTTTTATATCCCCATCACA3'; Next, we determined the chromosomal location of LINC02518 and predicted its secondary structure based on minimum free energy (MFE) and partition function (http://rna.tbi.univie.ac.at/). Additionally, IncLocator was used to predict subcellular localization of LINC02518 (13). Finally, the coding-potential of LINC02518 was evaluated by the following online metrics: PRIDE reprocessing 2.0, Lee translation initiation sites; PhyloCSF codon substitution frequency analysis; Coding Potential Assessment Tool (CPAT) coding probability, Bazzini small ORFs, and ORF finder software from the National Centre for Biotechnology Information.

\section{Follow-up}

All patients were followed up routinely by telephone, inpatient, and outpatient appointments after initial resection. Follow-up assessment included detailed history and physical examination, laboratory tests, and abdominal contrast-enhanced CT or MRI. Receiver operating characteristic (ROC) curves were generated using the LINC02518 levels in the 125 patients to determine the involvement of LINC02518 in diagnosing HCC. Patients were divided into two groups based on the relative expression of LINC02518 in tumor tissues: low and high expression groups. The overall survival time (OS) was calculated from the time of the initial resection until death or last follow-up. Disease-free survival (DFS) was calculated from the time of the initial resection until first intrahepatic recurrence, distant metastasis, death, or last followup. Relapse-free survival (RFS) was calculated from the time of the initial resection until first intrahepatic recurrence, death, or last follow-up. Metastases-free survival (MFS) was calculated from the time of the initial resection until extrahepatic metastasis, death, or last follow-up.

\section{Statistical analysis}

All the data were analyzed using SPSS 23.0 (version 23.0 for Windows; Armonk, NY; IBM Corp.), GraphPad Prism (version 6.01 for Windows, GraphPad software, La Jolla California, USA), and R 3.5.1. Independent-sample $t$ test was used to compare the difference in LINC02518 expression between paired samples. To determine significance between the baseline characteristics of two groups (sex, age, tumor size, number of tumor lesions, capsule, portal thrombosis, histologic grade, TNM stage, BCLC stage, ChildPugh, AFP, HBsAg, and cirrhosis), the continuity correction, Pearson $\chi 2$, and Fisher's exact tests were used. ROC curves were used to evaluate the diagnostic efficiency of LINC02518 in HCC. Kaplan-Meier plots were generated by the log-rank test to analyze OS, DFS, RFS, and MFS of patients with HCC. P $<0.05$ was considered statistically significant.

\section{Results}


LncRNA expression profiling of 125 pairs of $\mathrm{HCC}$ and corresponding non-tumor liver tissues in the discovery cohort by microarray revealed 218 differentially expressed IncRNAs (fold change $>1.25$; false discovery rate was set to 0 ). Of these, 85 and 133 were upregulated and downregulated in the HCC tissues, respectively; LINC02518 (ENSG00000232316.1) was selected for subsequent experiments as the novel IncRNA owing to its significant difference in expression between HCC and non-tumor tissues (Fig. 1a). qRT-PCR was also used to measure LINC02518 RNA in a cohort of 75 paired HCC and matched adjacent normal tissues. The LINC02518 RNA expression was significantly increased in tumor tissue compared with matched adjacent normal tissues(Fig. 1b).

LINC02518 is an intergenic IncRNA located on chromosome 6q21 with two exons encoding two major transcripts of 524 and $511 \mathrm{bp}$ (Fig. 2). The results of sequence alignment between the transcript variants of LINC02518 using APE software (http://biologylabs.utah.edu/jorgensen/wayned/ape/) are also shown in Fig. 2. Figure 3 shows the predicted secondary structure of LINC02518. The different online software indicate that LINC02518 is non-coding (Table 1). Table 2 lists the results obtained by IncLocator. LINC02518 was predicted to localize to the cytoplasm.

Table 1

The coding-potential of LINC02518 was evaluated by 6 kinds of online metrics.

\begin{tabular}{|lll|}
\hline Metric & Result & Interpretation \\
\hline PRIDE reprocessing 2.0 & 0 & non-coding \\
\hline Lee translation initiation sites & 0 & non-coding \\
\hline PhyloCSF score & -60.0596 & non-coding \\
\hline CPAT coding probability & $6.62 \%$ & non-coding \\
\hline Bazzini small ORFs & 0 & non-coding \\
\hline ORF Reader & -0.96 & non-coding \\
\hline
\end{tabular}

Table 2

The IncLocator Prediction Results of LINC02518

\begin{tabular}{|ll|}
\hline Subcellular locations & Score \\
\hline Cytoplasm & 0.76476840175 \\
\hline Nucleus & 0.0692847174226 \\
\hline Ribosome & 0.0296421878059 \\
\hline Cytosol & 0.117624789175 \\
\hline Exosome & 0.0186799038472 \\
\hline
\end{tabular}

\section{LINC02518 expression and HCC clinicopathological characteristics}


HCC patients were divided into high LINC02518-expressing and low LINC02518-expressing according to the optimal cutoff. The clinicopathological features showed that LINC02518 expression was not correlated with patient sex and age, tumor lesion numbers, capsule, portal thrombosis, histologic grade, Child-Pugh, AFP, HBsAg, and cirrhosis (all P > 0.05); however, LINC02518 expression was correlated with tumor size, TNM staging, and BCLC staging (both $\mathrm{P}<0.05$; Table 3). 
Table 3

Correlation between LINC02518 expression and clinicopathological characteristics of HCC

\begin{tabular}{|c|c|c|c|}
\hline \multirow[t]{2}{*}{ Clinical parameters } & \multicolumn{2}{|l|}{ LINC02518 } & \multirow[t]{2}{*}{$\mathbf{P}$} \\
\hline & Low expression $(n=95)$ & High expression $(n=30)$ & \\
\hline Age (ages) & & & 0.209 \\
\hline$<=50$ & 46 & 19 & \\
\hline$>50$ & 49 & 11 & \\
\hline Gender & & & 0.811 \\
\hline Male & 11 & 3 & \\
\hline Female & 84 & 27 & \\
\hline Size & & & $0.011^{*}$ \\
\hline$<=5 \mathrm{~cm}$ & 48 & 7 & \\
\hline$>5 \mathrm{~cm}$ & 47 & 23 & \\
\hline Lesion numbers & & & 0.071 \\
\hline Single & 69 & 16 & \\
\hline Two or more & 26 & 14 & \\
\hline Capsula & & & 0.184 \\
\hline No & 28 & 13 & \\
\hline Yes & 67 & 17 & \\
\hline Portal thrombsis & & & 0.124 \\
\hline No & 79 & 21 & \\
\hline Yes & 16 & 9 & \\
\hline Histologic grade & & & 0.563 \\
\hline Well & 3 & 0 & \\
\hline Moderately & 68 & 21 & \\
\hline Poorly & 24 & 9 & \\
\hline TNM stage & & & $0.033^{*}$ \\
\hline I & 56 & 10 & \\
\hline
\end{tabular}

*P values $<0.05$ were considered statistically significant. 


\begin{tabular}{|c|c|c|c|}
\hline \multirow[t]{2}{*}{ Clinical parameters } & \multicolumn{2}{|l|}{ LINC02518 } & \multirow[t]{2}{*}{$\mathbf{P}$} \\
\hline & Low expression $(n=95)$ & High expression $(n=30)$ & \\
\hline II & 17 & 11 & \\
\hline III & 22 & 9 & \\
\hline BCLC stage & & & $0.009 *$ \\
\hline A & 38 & 4 & \\
\hline B & 45 & 17 & \\
\hline C & 12 & 9 & \\
\hline Child-Pugh & & & 0.831 \\
\hline A & 91 & 29 & \\
\hline B & 4 & 1 & \\
\hline AFP & & & 0.240 \\
\hline$<=200 \mathrm{ng} / \mathrm{ml}$ & 59 & 15 & \\
\hline$>200 \mathrm{ng} / \mathrm{ml}$ & 36 & 15 & \\
\hline HBsAg & & & 0.546 \\
\hline Negative & 9 & 4 & \\
\hline Positive & 86 & 26 & \\
\hline Cirrhosis & & & 0.396 \\
\hline No & 42 & 10 & \\
\hline Yes & 53 & 20 & \\
\hline
\end{tabular}

Predictive value of LINC02518 for the diagnosis of HCC and the association between LINCO2518 expression and clinical outcomes in HCC patients.

The area under the ROC curve for LINC02518 expression in diagnosing HCC was 0.66 (95\% Cl: 0.59-0.72; Fig. 4). The median follow-up time was 55.3 months (range 3.0-105.0 months) for all the patients. At the end-point of the study, 49 (51.6\%) and 9 (30.0\%) patients in the low and high expression group, respectively were alive. The median and mean \pm standard deviation (SD) OS in patients were $54.5(95 \%$ Cl: 23.0, 85.9) and $55.9 \pm 4.0$ months, respectively. Kaplan-Meier survival analysis (Fig. 5a) revealed differences in the survival of the two groups of patients $(P=0.045)$ with a median survival of 65.1 months ( $95 \%$ Cl: NA, NA) for the low expression group compared to 15.9 months (95\% Cl: $0.1,31.7)$ for the high expression group. In DFS analysis, 35 (36.8\%) and 6 (20.0\%) patients in the low and high 
expression groups were in remission. The median and mean \pm SD DFS in patients were 19.8 (95\% Cl: 3.5, 36.1 ) and $42.7 \pm 4.0$ months, respectively. Kaplan-Meier survival analysis (Fig. $5 \mathrm{~b}$ ) showed that DFS between the patient groups was significant $(P=0.042)$ with a median DFS of 36.8 months $(95 \% \mathrm{Cl}: 14.9$, 58.8) for the low expression group compared to 10.0 months (95\% Cl: 7.1, 12.8) for the high expression group. In RFS analysis, 37 (38.9\%) and 7 (23.3\%) patients in the low and high expression group were of relapse-free status. The median and mean \pm SD RFS in the patients were 26.1 months ( $95 \%$ Cl: 9.8, 42.4) and $44.5 \pm 4.0$ months, respectively. Kaplan-Meier survival analysis (Fig. $5 \mathrm{c}$ ) showed difference between the RFS of the two patient groups $(P=0.036)$ with a median RFS of 37.3 months $(95 \% \mathrm{Cl}: 19.1,55.5)$ for the low expression group compared to 9.8 months $(95 \% \mathrm{Cl}: 7.0,12.6)$ for the high expression group. In MFS analysis, $47(49.5 \%)$ and $8(26.7 \%)$ patients in the low and high expression group were still devoid of metastasis. The median and mean \pm SD DFS of the patients were 37.3 months $(95 \% \mathrm{Cl}: 10.2,64.4)$ and $53.0 \pm 4.1$ months, respectively. Kaplan-Meier survival analysis (Fig. $5 \mathrm{~d}$ ) showed a significant difference in the MFS between the patient groups $(P=0.039)$ with a median MFS of 57.5 months $(95 \% \mathrm{Cl}: 30.4$, 84.6) for the low expression group compared to 14.7 months (95\% Cl: 9.1, 20.2) for the high expression group. Thus, LINC02518 expression was correlated with poor prognosis of HCC.

\section{Discussion}

$\mathrm{HCC}$ is one of the leading causes of lethality worldwide. Although there has been an increase in the rate of survival of HCC patients with the advances in medical technology, the molecular pathogenesis of HCC and genetic factor responsible remain to be understood $(2,14-16)$. There has been limited success in improving DFS of HCC patients. Thus, novel cancer-promoting genes involved in HCC must be identified and characterized to obtain a better understanding of this lethal disease and develop therapeutic interventions (14). In this study, we identified a novel differentially expressed IncRNA, LINC02518, located on chromosome 6q21. LINC02518 was overexpressed in HCC tissues and positively correlated with the clinicopathological features of HCC. Importantly, higher LINC02518 levels predicted lower OS, DFS, RFS, and MFS in patients with HCC, suggesting that LINC02518 may be a promising prognostic biomarker for HCC.

The IncRNA expression profiles of HCC identified by microarray analysis have been previously reported $(17,18)$. Zhu et al. performed an IncRNA gene expression profile analysis in 3 pairs of human HCC and adjacent non-tumor tissues using microarray containing 33,045 IncRNAs and 30,215 coding transcripts and identified 214 IncRNAs and 338 mRNAs abnormally expressed in all three HCC tissues with the genome-wide IncRNAs and mRNAs expression profile analysis(17). Additionally, Xu et al. collected nine microarray expression data sets of mRNAs, miRNAs, and IncRNAs associated with HCC and found that 628 mRNAs, 15 miRNAs, and 49 IncRNAs were differentially expressed using integrative analysis(18). In our study, we analyzed IncRNA expression profiles of 125 HCCs with a custom microarray having a total of 2,412 IncRNA transcripts, which might significantly reduce the confounding factors caused by individual sample differences. Also, the number and probes of IncRNA transcripts in our study were different from those in the above studies; therefore, further integrative analysis is warranted to identify additional IncRNA candidates. 
The differential expression of IncRNAs in HCC is correlated with the prognosis of the disease. IncRNAs, such as MVIH, MALAT-1, HULC, InCRNA-ATB, and LINC01128 are present in low amounts in normal liver cells and are upregulated in HCC $(14,19-22)$. Some IncRNAs are downregulated in HCC samples, such as PSTAR, MT1DP, and DILC (23-25). These IncRNAs may serve as tumor markers for the early diagnosis of HCC. The expression of these IncRNAs was also closely associated with the prognosis of patients with $\mathrm{HCC}$ and its clinicopathological features. Li et al. analyzed the correlation between LINC01138 levels and clinicopathological features in $120 \mathrm{HCC}$ and adjacent non-cancerous tissues; LINC01138 is overexpressed in $53.3 \%$ of the HCC samples and its expression is positively correlated with tumor size, AFP levels, and hepatitis B surface antigen (HBsAg) levels (14). High LINC01138 levels are associated with poor OS in HCC patients (14). Previous studies have shown that some IncRNAs are downregulated in HCC tissues, such as p53-stabilizing and activating RNA (PSTAR) (23). PSTAR helps accumulate and transactivate p53; low levels of PSTAR predict poor OS and RFS in patients with HCC, especially those with wild-type p53 (23). In this study, LINC02518 expression was significantly upregulated in HCC samples as compared with that of adjacent non-cancerous tissues; LINC02518 expression was also correlated with tumor size, TNM staging, and BCLC staging. HCC patients with high LINC02518 levels resulted in lower RFS, MFS, and OS than those with low LINC02518 expression.

LncRNAs regulate gene expression at various levels, including chromatin modification, transcription, and posttranscriptional processing (26). Firstly, the interaction between IncRNAs and proteins modulate protein activity and localization. Secondly, IncRNAs could be processed into small, single- or doublestranded RNAs that could act as endo-siRNAs targeting other RNAs, leading to target degradation. LncRNAs can act as an "miRNA sponge" and sequester miRNAs to inactivate small regulatory RNAs that influence the expression of miRNA target genes. Furthermore, IncRNAs regulate transcription via recruiting transcription factors to their target gene promoters, thereby activating gene expression. However, they can also inhibit the binding of general transcription factors, potentially by forming RNADNA-triplexes. Finally, IncRNAs contribute to transcriptome complexity, as they can regulate alternative splicing of pre-mRNAs. The balance between transcriptionally active euchromatin and silent heterochromatin is controlled by IncRNAs. They can interact with chromatin remodeling complexes and induce local or global changes in chromatin packaging (26). However, the mechanism underlying the role of LINC02518 in HCC remains unclear. Previous studies have found that IncRNA localizes to the cytoplasm, suggesting a possible function as a competitive endogenous RNA acting as a molecular sponge for miRNAs. For example, IncRNA HOXD-AS1 is a competitive endogenous RNA that facilitates liver cancer metastasis by regulating SOX4 (27) and IncRNA MCM3AP-AS1 promotes the growth of HCC by targeting the miR-194-5p/FOXA1 axis (28). In this study, LINC02518 primarily localized to the cytoplasm, indicating a function as an "miRNA sponge" that sequesters miRNAs to inactivate small regulatory RNAs. However, the mechanisms underlying the role of LINC02518 as a competitive endogenous RNA need to be explored.

In conclusion, this study demonstrates that LINC02518 expression is negatively correlated with HCC prognosis. The discovery of LINC02518, a promising prognostic indicator, provides insight into hepatic carcinogenesis and may facilitate the development of approaches for cancer screening and treatment. 
Therefore, decreasing the expression of LINC02518 might be an effective approach for suppressing the development, metastasis, and prognosis of HCC. However, our study had a limited sample size. Thus, studies with a large sample size need to be performed for a more comprehensive understanding of the clinical potential of our findings.

\section{List Of Abbreviations}

AFP, a-fetoprotein; CT, computed tomography; DFS, disease-free survival; HCC, hepatocellular carcinoma; MFS, metastases-free survival; MR, magnetic resonance imaging; RFS, relapse-free survival; ROC, receiver operating characteristic; OS, overall survival

\section{Declarations}

\section{Ethics approval and consent to participate}

The study involving patients with unresectable hepatocellular carcinoma was reviewed and approved by the ethics committee of our hospital. Written informed consent was waived because the study was retrospective.

Consent for publication: Not Applicable.

\section{Availability of data and materials}

The datasets analyzed in this article are not publicly available. Requests to access the datasets should be directed to Jiaping Li, jiapingli3s@126.com.

Competing interests: The authors declare that they have no competing interests.

Funding: None.

\section{Authors' contributions}

CONCEPTION: Wei Cui, Jiaping Li,

INTERPRETATION OR ANALYSIS OF DATA: Wei Cui, Jingzhi Huang,Ruiqi Wang, Rongde Xu, Feng Shi PREPARATION OF THE MANUSCRIPT:Wei Cui, Jingzhi Huang, Yu Wang, Xiaoming Chen, Jiaping Li, Rongde Xu

REVISION FOR IMPORTANT INTELECTUAL CONTENT: Wei Cui, Jingzhi Huang, Jiaping Li, Rongde Xu SUPERVISION:Wei Cui, Jiaping Li, Rongde Xu

\section{Acknowledgements}

We thank Prof. Hunyun Wang from State Key Laboratory of Oncology in South China, Sun Yat-Sen University Cancer Center for providing the standardized data of IncRNA microarray (GEO GSE72170). We thank all the patients, clinicians, and support staff who participated in this study. 


\section{References}

1. Villanueva A. Hepatocellular Carcinoma. New Engl J Med. 2019;380(15):1450-62.

2. Q G, H Z, L D, W S, R C, Z S, et al. Integrated Proteogenomic Characterization of HBV-Related Hepatocellular Carcinoma. Cell. 2019;179(2):561-77.e22.

3. National Comprehensive Cancer Network. Hepatobiliary Cancers $\mathbb{N} C C N$ Clinical Practice Guidelines in Oncology 2019.V3】. 2019.

4. Yang F, Zhang L, Huo XS, Yuan JH, Xu D, Yuan SX, et al. Long noncoding RNA high expression in hepatocellular carcinoma facilitates tumor growth through enhancer of zeste homolog 2 in humans. Hepatology. 2011;54(5):1679-89.

5. Schmitt AM, Chang HY. Long Noncoding RNAs in Cancer Pathways. Cancer Cell. 2016;29(4):452-63.

6. Sahu A, Singhal U, Chinnaiyan AM. Long noncoding RNAs in cancer: from function to translation. Trends Cancer. 2015;1(2):93-109.

7. Atianand MK, Caffrey DR, Fitzgerald KA. Immunobiology of Long Noncoding RNAs. Annu Rev Immunol. 2017;35:177-98.

8. Yang Y, Chen L, Gu J, Zhang H, Yuan J, Lian Q, et al. Recurrently deregulated IncRNAs in hepatocellular carcinoma. Nat Commun. 2017;8:14421.

9. European Association for the Study of the Liver. Electronic address eee, European Association for the Study of the L. EASL Clinical Practice Guidelines: Management of hepatocellular carcinoma. J Hepatol. 2018;69(1):182-236.

10. Forner A, Reig ME, de Lope CR, Bruix J. Current strategy for staging and treatment: the BCLC update and future prospects. Semin Liver Dis. 2010;30(1):61-74.

11. Edge SB BD, Compton CC, Fritz AG, Greene FL, Trotti A. AJCC cancer staging manual. 7th ed. New York. Springer. 2010:191-200.

12. Chen DL, Lu YX, Zhang JX, Wei XL, Wang F, Zeng ZL, et al. Long non-coding RNA UICLM promotes colorectal cancer liver metastasis by acting as a ceRNA for microRNA-215 to regulate ZEB2 expression. \#N/A. 2017;7(19):4836-49.

13. Z C, X P, Y Y, Y H, HB S. The IncLocator: a subcellular localization predictor for long non-coding RNAs based on a stacked ensemble classifier. Bioinformatics. 2018;34(13):2185-94.

14. Z L, J Z, X L, S L, Q W, Chen D, et al. The LINC01138 drives malignancies via activating arginine methyltransferase 5 in hepatocellular carcinoma. Nat Commun. 2018;9(1):1572.

15. Zhang J, Lin H, Wang XY, Zhang DQ, Chen JX, Zhuang Y, et al. Predictive value of microRNA-143 in evaluating the prognosis of patients with hepatocellular carcinoma. Cancer Biomark. 2017;19(3):257-62.

16. CM W, FH T, IO N. Non-coding RNAs in hepatocellular carcinoma: molecular functions and pathological implications. Nat Rev Gastroenterol Hepatol. 2018;15(3):137-51. 
17. Zhu J, Liu S, Ye F, Shen Y, Tie Y, Zhu J, et al. The long noncoding RNA expression profile of hepatocellular carcinoma identified by microarray analysis. PLoS One. 2014;9(7):e101707.

18. Xu J-H, Chang W-H, Fu H-W, Yuan T, Chen P. The mRNA, miRNA and IncRNA networks in hepatocellular carcinoma: An integrative transcriptomic analysis from Gene Expression Omnibus. Mol Med Report. 2018;17(5):6472-82.

19. Chen L, Yao H, Wang K, Liu X. Long Non-Coding RNA MALAT1 Regulates ZEB1 Expression by Sponging miR-143-3p and Promotes Hepatocellular Carcinoma Progression. J Cell Biochem. 2017;118(12):4836-43.

20. SX Y, F Y, Y Y, QF T, J Z, G H, et al. Long noncoding RNA associated with microvascular invasion in hepatocellular carcinoma promotes angiogenesis and serves as a predictor for hepatocellular carcinoma patients' poor recurrence-free survival after hepatectomy. Hepatology. 2012;56(6):223141.

21. H Z, Z L, F L, C S, H Z, Y L, et al. Long noncoding RNA HULC promotes hepatocellular carcinoma progression. Aging (Albany NY). 2019;11(20):9111-27.

22. JH Y, F Y, F W, JZ M, YJ G, QF T, et al. A long noncoding RNA activated by TGF- $\beta$ promotes the invasion-metastasis cascade in hepatocellular carcinoma. Cancer Cell. 2014;25(5):666-81.

23. G Q, X T, H L, P C, X C, J S, et al. Long Noncoding RNA p53-Stabilizing and Activating RNA Promotes p53 Signaling by Inhibiting Heterogeneous Nuclear Ribonucleoprotein K deSUMOylation and Suppresses Hepatocellular Carcinoma. Hepatology. 2020;71(1):112-29.

24. Yu W, Qiao Y, Tang X, Ma L, Wang Y, Zhang X, et al. Tumor suppressor long non-coding RNA, MT1DP is negatively regulated by YAP and Runx 2 to inhibit FoxA1 in liver cancer cells. Cell Signal. 2014;26(12):2961-8.

25. Wang $X$, Sun W, Shen W, Xia M, Chen C, Xiang D, et al. Long non-coding RNA DILC regulates liver cancer stem cells via IL-6/STAT3 axis. J Hepatol. 2016;64(6):1283-94.

26. Gutschner T, Diederichs $S$. The hallmarks of cancer: a long non-coding RNA point of view. RNA Biol. 2012;9(6):703-19.

27. Wang H, Huo X, Yang XR, He J, Cheng L, Wang N, et al. STAT3-mediated upregulation of IncRNA HOXD-AS1 as a ceRNA facilitates liver cancer metastasis by regulating SOX4. Mol Cancer. 2017;16(1):136.

28. Wang Y, Yang L, Chen T, Liu X, Guo Y, Zhu Q, et al. A novel IncRNA MCM3AP-AS1 promotes the growth of hepatocellular carcinoma by targeting miR-194-5p/FOXA1 axis. Mol Cancer. 2019;18(1):28.

\section{Figures}



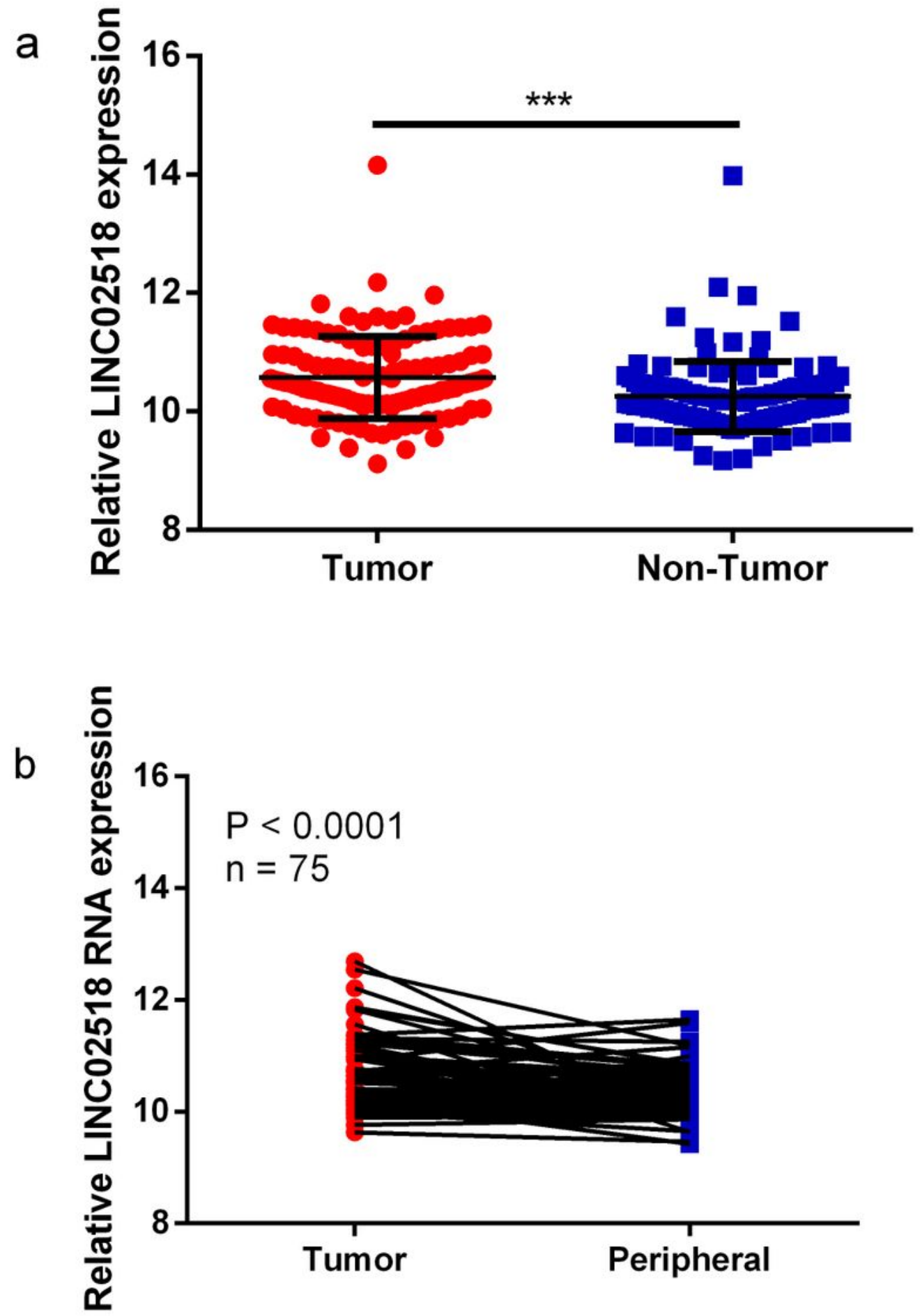

Figure 1

LINC02518 is upregulated in human HCC tissues. a. The relative LINC02518 expression in 125 pairs of HCC and corresponding nontumor liver tissues. b. The relative RNA levels of LINC02518 by qRT-PCR were higher in tumor tissues compared with adjacent non-tumorous tissues. Note: ${ }^{\star \star}, \mathrm{P}<0.001$ 
$6 q 21$

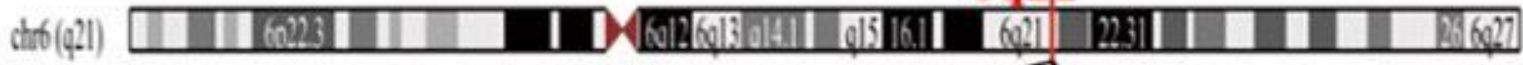

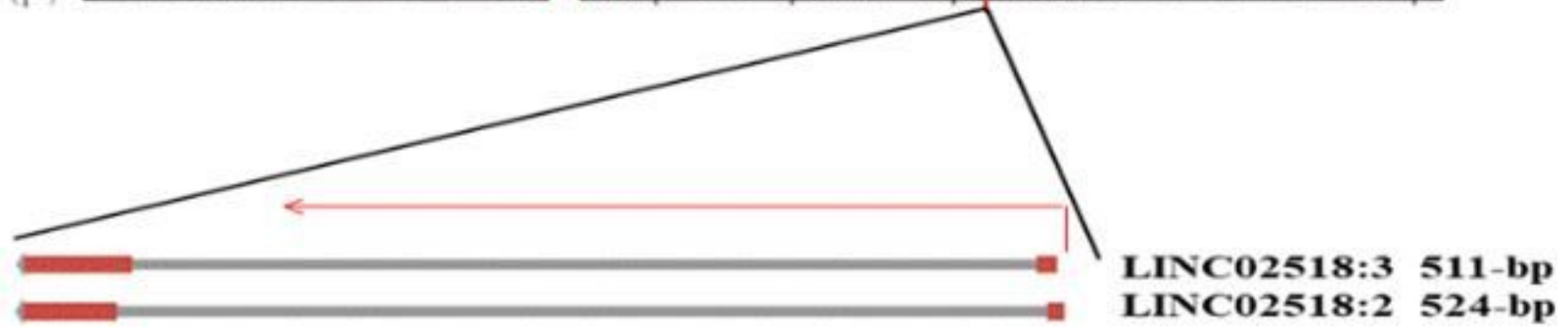

5' 1 CTGAGG-AGCTAT-CAAAAACTGCTTGCCATCTT-TCCAAATCATCTCTGTCTGCAGTGA--CCATGTCTTAATCCTGAAGGAAAACAA---GGTGGAAA 92

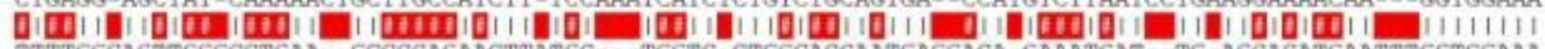

1 TITTGGCAGTTGGGCTGAA--GCCCCAGAACTTATGC--TGGC-CTGGCACCAATGAGGACA-GAAATGAT-TG-AGCACATGAATTTGGTGGAAA 90

93 TTGATTTTTACTTCTCTGCAGCTAAGTATGTGGCAGTCTTGGCCACATGGTGACTCAGCAGAGGAGCCTATGTACTGGCTGTCCTGATGCTCTTCCTGAT 192 11111111111111111111111111111111111111111111111111111111111111111111111111111111111111111111111111111

91 TTGATTTTTACTTCTCTGCAGCTAAGTATGTGGCAGTCTTGGCCACATGGTGACTCAGCAGAGGAGCCTATGTACTGGCTGTCCTGATGCTCTTCCTGAT 190

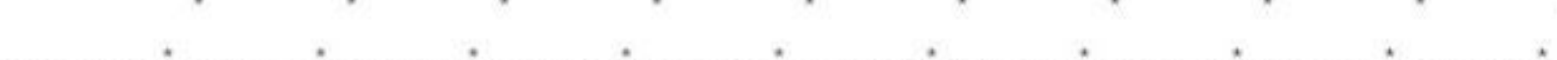

493 AAAAGCCAATGAAAGATATCATTAATTGTTAA $524 \quad$ 3'

491 AAAAGCCAATGAAAGATATCA N 511

\section{Figure 2}

Schematic representation of exons and transcripts of LINC02518 with sequence alignment and its loci on human chromosome 6q21 in UCSC Genome browser (GRCh37/hg38). 

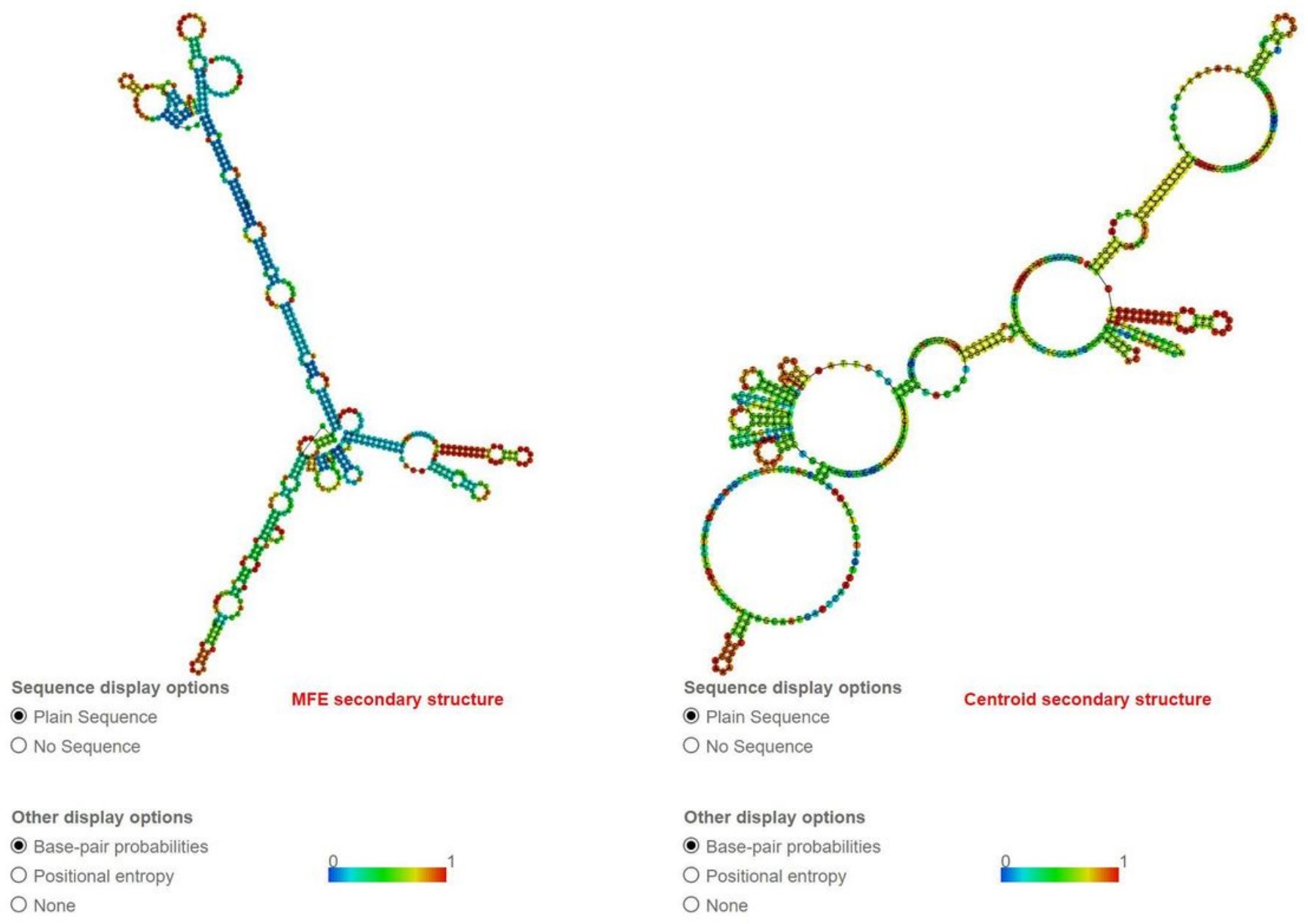

Figure 3

Prediction of LINC02518 structure based on minimum free energy (MFE) and partition function. Color scale indicates the confidence for the prediction for each base with shades of red indicating strong confidence (http://rna.tbi.univie.ac.at/). 


\section{Performance of LINC02518}

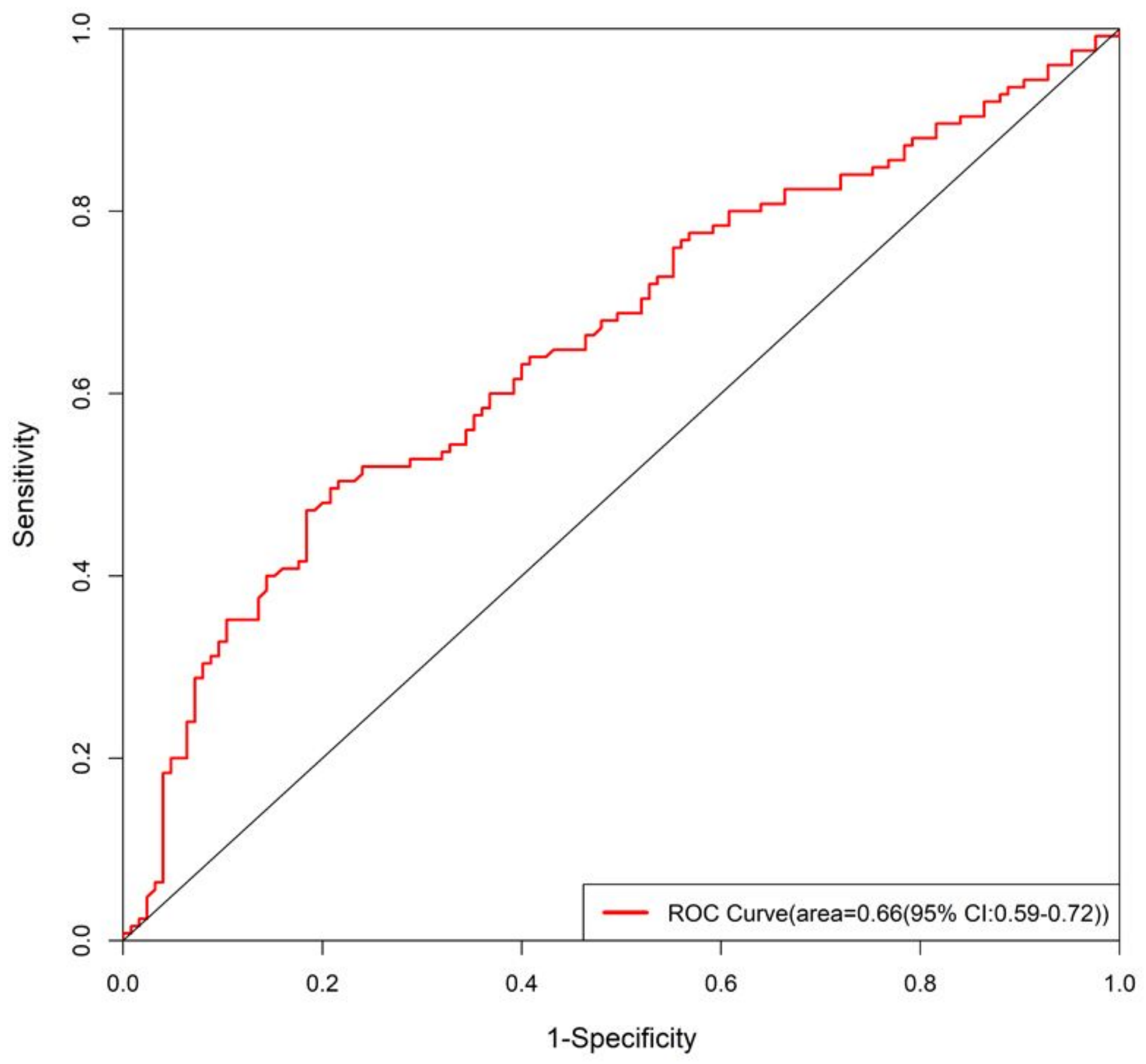

Figure 4

ROC curve of LINC02518 for the diagnosis of hepatocellular carcinoma. Note: ROC, receiver operating characteristic 

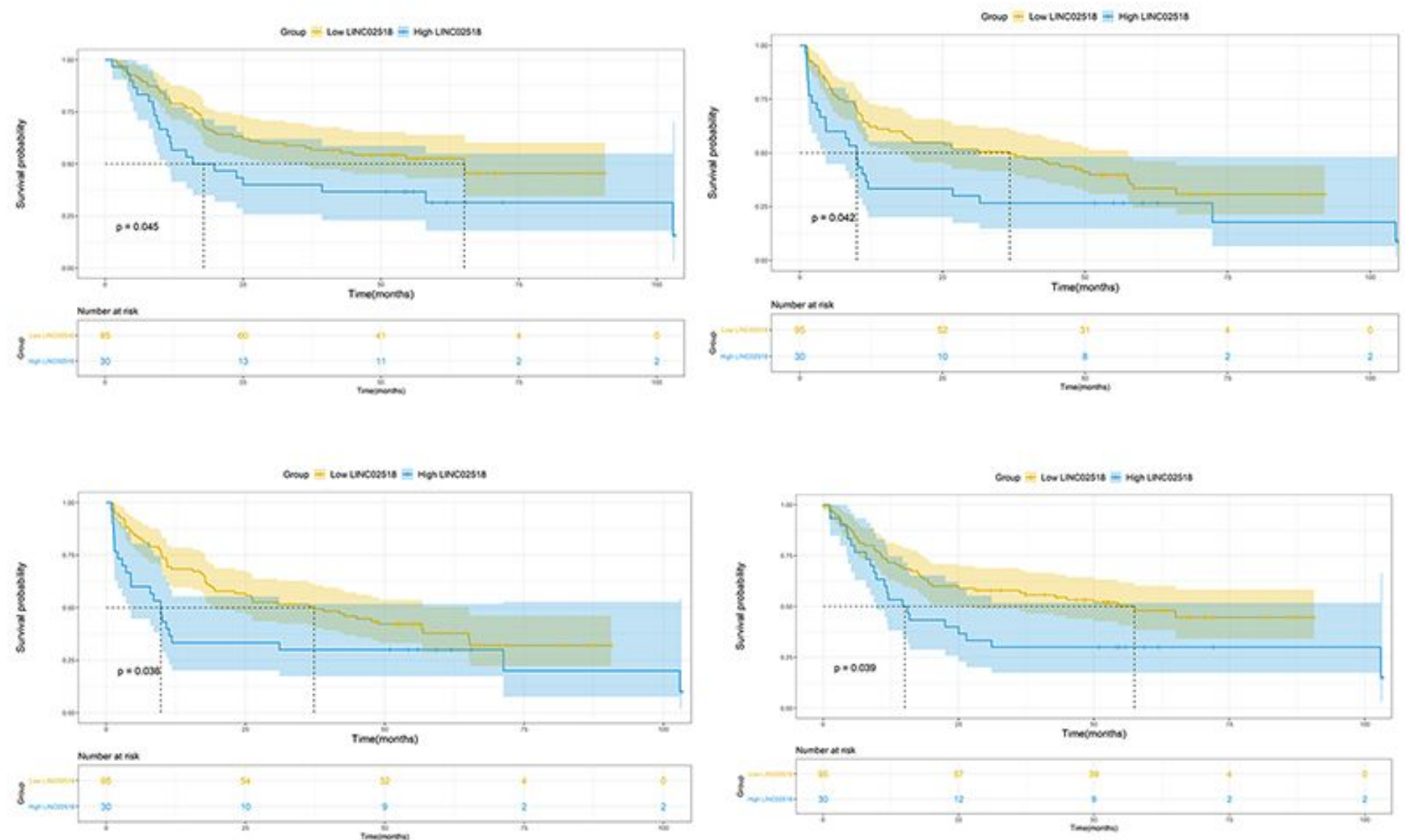

Figure 5

Clinical outcomes of $125 \mathrm{HCC}$ patients who underwent liver surgery were compared between the LINC02518 high-expression and LINC02518 low-expression groups a. OS b. DFS c. RFS d.MFS 